\title{
Modelling of the Marine Traffic Situation Complexity
}

\author{
Xiaoqiao Geng ${ }^{1,2}$, Liang Huang ${ }^{1,2}$, Yuanqiao Wen ${ }^{1,2}$ and Chunhui Zhou ${ }^{1,2}$ \\ ${ }^{1}$ School of navigation, Wuhan University of Technology, Wuhan, 430063, China \\ ${ }^{2}$ Hubei Key Laboratory of Inland Shipping Technology, Wuhan, 430063, China
}

\begin{abstract}
With the growth of traffic density, traditional collision risk models cannot thoroughly describe the complex traffic any more. Therefore, in this paper, a marine traffic situation complexity model, which is based on the relationship between ships, is introduced to metric the complexity of vessel traffic. Firstly, traffic density factor and traffic conflict factors are considered to construct the traffic unit complexity model, reflecting the traffic complexity and traffic characteristics; Secondly, by extending the traffic unit complexity model into the own-ship complexity model, the influences of the positions of other ship on the complexity spatial distribution are analysed; Finally, two scenarios are imported to validate the availability of the complexity model. The verification results show that the complexity can respond to different route structures and traffic situations sensitively.
\end{abstract}

\section{Introduction}

The tendencies of the marine traffic density increasing has increased the difficulty of water traffic management. To make up for the limitations of traditional risk models that most of them only use DCPA and TCPA as the evaluation indicators [1], which only measure the spatial relationships and urgency degree of two ships, without considering their relative position, marine traffic complexity is introduced to describe the complicated situation of marine traffic. There have been some progresses in complexity researching in the fields of air transportation and road traffic: One the one hand, researchers use linear[2] and nonlinear[3] modelling methods to construct the dynamic density model of airspace complexity.[4] On the other hand, researchers think complexity can be considered as the subjective description of traffic situation using intrinsic properties[5]. Airspace complexity model was established based on the coupling relationship of traffic flow.[6,7] Therefore, it is needed to research into the traffic flow complexity in the marine traffic field. In this paper, intrinsic properties complexity is used to research the marine traffic situation, and two complexity factors of traffic density and traffic conflict are proposed to construct the complexity model, then verification is made.

\section{Model of marine traffic situation complexity}

In this paper, traffic situation complexity, which is related with the traffic flow characteristics is researched. Traffic flow is characterized by the vessel traffic density as well as the geometrical relationships between vessels.
Therefore, researches on the traffic situation complexity can be carried from vessel traffic density and geometrical relationships between vessels. In the following research, the traffic relationship unit $V C_{i j}$ (Vessel Couple) constructed by two ships of $i$ and $j$ is defined.

\subsection{Influential factors of traffic density}

Definition 1: Traffic complexity directly caused by the traffic density is called as complicacy I $C_{\text {First } i j \text {. Traffic }}$ density described by the ships amount in unit water area can't fully represent the traffic complicacy, it is also related with the space among ships and the distribution of ships.

Definition2: The traffic density in the traffic unit is related with the relative distance of ships, and it equals the value of complicacy I $C_{F i v s t i j}$. Then there is:

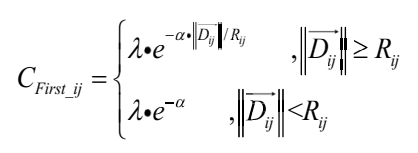

In Eq. $1, \alpha, \lambda$ are correction parameters. $R_{i j}$ is the safe distance within $V C_{i j} \cdot\left\|\overrightarrow{D_{i j}}\right\|$ is the relative distance between ship $i$ and $j . C_{\text {First } i j}$ is in nonlinear increase with the decrease of $\left\|\overrightarrow{D_{i j}}\right\|$.

\subsection{Influential factor of traffic conflict}

Definition 3: Traffic complexity caused by the traffic situation in the traffic unit is defined as the complexity factorII, which is called as conflict factor $C_{\text {Second } i j}$. And $C_{\text {Second } i j}=\psi\left(\overrightarrow{D_{i j}}, \overrightarrow{v_{i j}}, \theta\right), \overrightarrow{D_{i j}}$ is the relative distance, $\overrightarrow{v_{i j}}$ is 
the relative speed, $\theta$ is the track-crossing angle. In this paper, the process of traffic conflict is divided into four phases: judgement of converging tendency, estimation of converging consequence, measurement of urgency degree and difficulty of risk elimination.

\subsubsection{Converging situations}

When the relative distance of two ships is decreasing, then there is converging tendency:

$$
d\left\|\overrightarrow{D_{i j}}\right\| / d t=\left\|\overrightarrow{v_{i j}}\right\| \cdot \cos \left(\overrightarrow{v_{i j}}, \overrightarrow{D_{i j}}\right)=\overrightarrow{v_{i j}} \cdot \overrightarrow{D_{i j}} /\left\|\overrightarrow{D_{i j}}\right\|
$$

In Fig. 2, $\alpha$ is the angle between $\overrightarrow{v_{i j}}$ and $\overrightarrow{D_{i j}}$. When $\alpha \in(90,180], \cos \left(\overrightarrow{v_{i j}}, \overrightarrow{D_{i j}}\right)<0$, then the two ships have converging tendency(shown in Fig. 1(A)), and there exists traffic conflict; when $\alpha \in[0,90], \cos \left(\overrightarrow{v_{i j}}, \overrightarrow{D_{i j}}\right) \geq 0$, then the two ships have separating tendency(shown in fig.1(B)), and there is no traffic conflict. Defining function $\mathrm{mix}_{i j}$ to judge whether there exists converging tendency within $V C_{i j}$, then there is:

$$
C_{m i x i j}=\ell_{\mathrm{R}}\left\{\overrightarrow{v_{i j}} \cdot \overrightarrow{D_{i j}} /\left\|\overrightarrow{D_{i j}}\right\|\right\}= \begin{cases}1, & \overrightarrow{v_{i j}} \cdot \overrightarrow{D_{i j}} /\left\|\overrightarrow{D_{i j}}\right\|<0 \\ 0, & \overrightarrow{v_{i j}} \cdot \overrightarrow{D_{i j}} /\left\|\overrightarrow{D_{i j}}\right\| \geq 0\end{cases}
$$

\subsubsection{Converging consequence}

DCPA (Distance to Closest Point of Approach) is used to measure the dangerous degree: When there is no converging tendency then there is no potential collision risk between these two ships; when there is converging tendency, then there exists traffic conflict. Using $C_{\text {Latent } i j}$ to represent the converging consequences of two ships within $V C_{i j}$, then the following equation can be established:

$$
\begin{aligned}
& C_{\text {Latent } \_i j}=\varepsilon\left(D_{C P A},\left\|\overrightarrow{D_{i j}}\right\|\right)=\lambda \cdot\left(e^{-\alpha \cdot D_{C P A} / R_{i j}}-e^{-\alpha \cdot\left\|\overrightarrow{D_{i j}}\right\| / R_{i j}}\right) \\
& C_{\text {mix }_{-} i j}=1
\end{aligned}
$$

In Eq. 4, $D C P A=\left\|\overrightarrow{D_{i j}}\right\| \cdot \sin (\pi-\alpha)=\left\|\overrightarrow{D_{i j}}\right\| \cdot \sin (\alpha) ; \quad \alpha$ is the angle between $\overrightarrow{D_{i j}}$ and $\overrightarrow{D_{i j}} ; \alpha=\arccos \left(\overrightarrow{v_{i j}} \cdot \overrightarrow{D_{i j}} /\left\|\overrightarrow{v_{i j}}\right\| \mid \overrightarrow{D_{i j}} \|\right)$.

\subsubsection{Urgency degree}

In navigational practice, TCPA(Time to Closest Point of Approach)is used to represent the urgency degree of two converging ships. And $T_{C P A}=\left\|\overrightarrow{D_{i j}}\right\| \cdot \cos (\alpha) /\left\|\overrightarrow{v_{i j}}\right\|=\overrightarrow{D_{i j}} \cdot \overrightarrow{v_{i j}} /\left\|\overrightarrow{v_{i j}}\right\|^{2}$. $T_{C R}$ (Time Collision Risk) is also introduced to measure the urgency degree of two ships, then there is:

$$
\begin{aligned}
T_{C R_{-} i j} & =\tau\left(\overrightarrow{D_{i j}}, \overrightarrow{v_{i j}}\right) \\
& = \begin{cases}1, & T_{C P A} \leq t_{1} \\
{\left[\left(t_{2}-T_{C P A}\right) /\left(t_{2}-t_{1}\right)\right]^{3.03},} & t_{1}<T_{C P A} \leq t_{2} \\
0, & T_{C P A}>t_{2} \text { or } T_{C P A}<0\end{cases}
\end{aligned}
$$

In Eq.5, $t_{2}=\sqrt{12^{2}-D_{C P A}{ }^{2}} /\left\|\overrightarrow{v_{i j}}\right\|, t_{1}=\sqrt{R_{i j}{ }^{2}-D_{C P A}{ }^{2}} /\left\|\overrightarrow{v_{i j}}\right\| ;$ $R_{i j}$ is the radius of the security domain of ship $i$.

\subsubsection{Difficulty degree of risk elimination}

In navigational practice, the difficulty of collision risk elimination is not in linear relationship with the trackcrossing angle. Using $C_{\text {angle } i j}$ to represent the difficulty degree of risk elimination in the condition of different track-crossing angle $\theta$, their relationship is shown in Fig. 2.

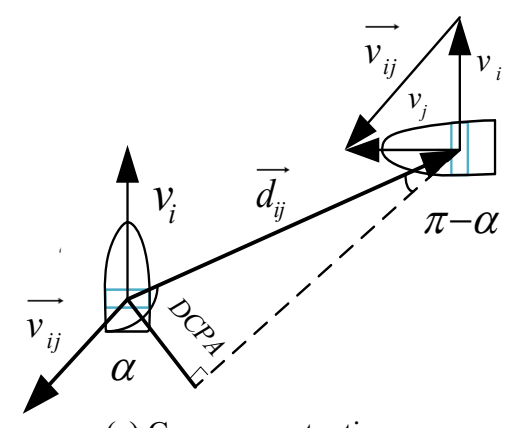

(a) Converge sutuation

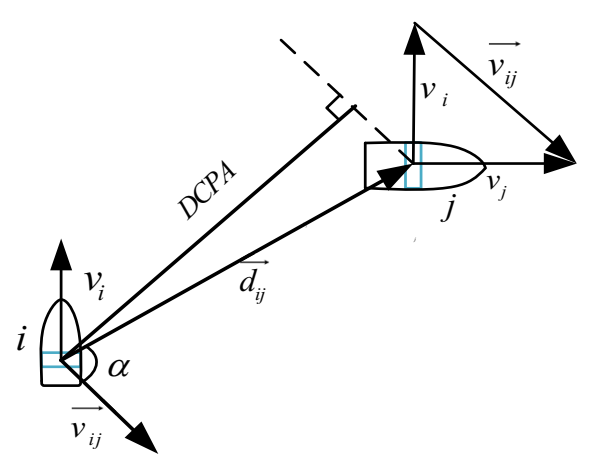

(b) Dissociate situation

Figure 1. Converge and dissociate situation.

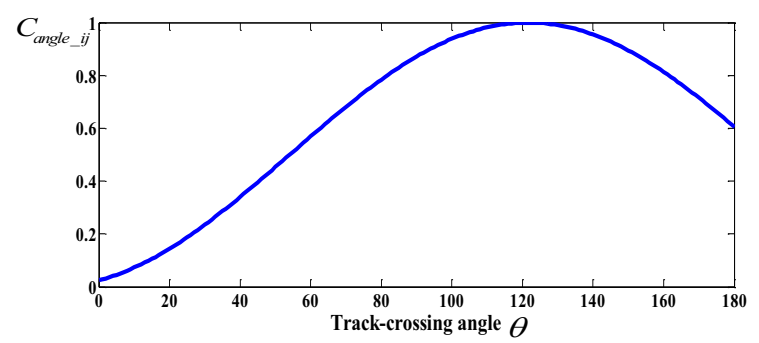

Figure 2. Difficulty of risk elimination in different angles.

And the mathematical expression is as follows:

$$
C_{\text {angle } i j}=f(\theta)=\frac{1}{2} \cdot\left\{1-\cos \left[\left(\frac{180}{67.5}\right) \cdot \frac{\theta}{2} \cdot \frac{\pi}{180}+\frac{\pi}{10}\right]\right\} \text { (6) }
$$

From Fig. 2, we can see that on the condition that $\theta \in(0,120]$,difficulty degree of risk elimination increases and reaches its highest when $\theta=120$; while on the condition that $\theta \in(120,180]$, it decreases. And there is $C_{\text {angle } i j} \in(0,1]$. In conclusion, the complexity increment of $C_{\text {Second } \_i j}$ caused by traffic conflicts can be measured as follows: $C_{m i x} i j$ is used to judge whether traffic conflict 
exists within the traffic unit; $C_{\text {Latent } \_i j}$ is used to describe the complexity increment without any action taken. $T_{C R} i j$ is used to describe the urgency degree of the collision risk. $C_{\text {angle } i j}$ is used to describe the difficulty of collision risks elimination. Therefore, $C_{\text {Second_ij }}$ can be expressed as Eq.7.

$$
\begin{aligned}
& C_{\text {Second } \_j_{j}}=\psi\left(\overrightarrow{D_{i j}}, \overrightarrow{v_{i j}}, \theta\right) \\
& =C_{\text {mix } i j} \cdot C_{\text {Latent_ij }} \cdot T_{C R_{-} i j} \cdot C_{\text {angle } i j} \\
& =l_{\mathrm{R}^{-}}\left\{\frac{\overrightarrow{v_{i j}} \cdot \overrightarrow{D_{i j}}}{\left\|\overrightarrow{D_{i j}}\right\|}\right\} \cdot \varepsilon\left(\overrightarrow{D_{i j}}, \overrightarrow{v_{i j}}\right) \cdot \tau\left(\overrightarrow{D_{i j}}, \overrightarrow{v_{i j}}\right) \cdot f(\theta)
\end{aligned}
$$

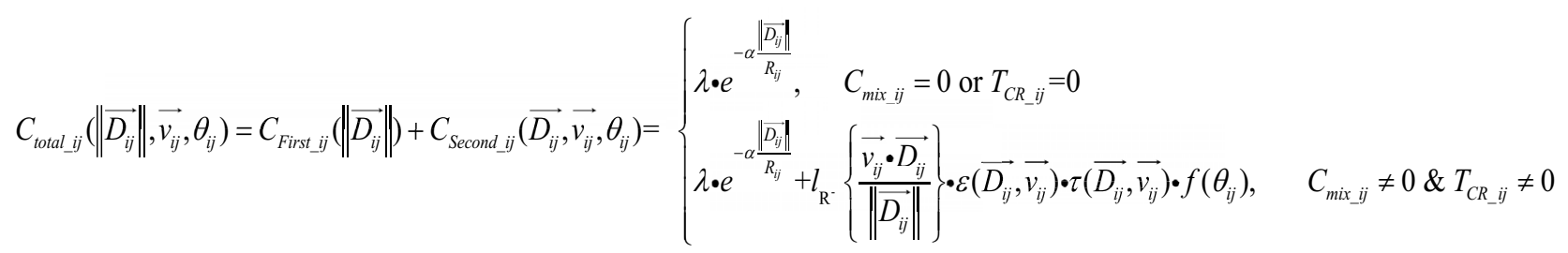

\subsection{Complexity model}

The traffic situation complexity of $V C_{i j}$ consists of complicacy I and complicacy II. Therefore, it can be defined as $C_{\text {total } \_i j}$ in the adding format (Eq.8). And supposing that when the distance between two ships is less than $0.50 \mathrm{nmile}$, the complexity reaches its maximum value, which is set as 80 ; when it decreases to 3 nmile, corresponding actions should be taken and the complexity value is 0.25 times of the maximum one. Therefore, parameters can be set as: $\alpha=2 \cdot \ln (2) / 5, \lambda=80 \times 2^{2 / 5}$.
Complexity of the traffic unit can be calculated using Eq.8, then the distribution pattern of complexity, which can be applied to collision risk elimination, can be acquired using the interpolation formula. Supposing $i$ as the own-ship, then in the perspective of $\operatorname{ship} i$, recognize all the traffic units $V C_{i j}$ concluding ship $i$, where, $j \in(1: n),(j \neq i)$. The complexity value of ship $j$ can be calculated as $C_{\text {total } i j}\left(\overrightarrow{D_{i j}}, \overrightarrow{v_{i j}}, \theta_{i j}\right)$. Then meshing is made to the area, and Eq.9 is used to calculate the complexity value of each grid. Finally, the spatial distribution pattern of this water area can be acquired.

$$
C(a, b)=\sum_{j}^{n} \frac{C_{\text {total_ij }}\left(\overrightarrow{D_{i j}}, \overrightarrow{v_{i j}}, \theta_{i j}\right)}{d_{j}{ }^{3}} \bullet \sum_{j}^{n} d_{j}{ }^{3}
$$

In Eq.9, $a, b$ represent the row and column number respectively after the meshing process; $C_{\text {total } \_i j}\left(\overrightarrow{D_{i j}}, \overrightarrow{v_{i j}}, \theta_{i j}\right)$ represents the complexity value of ship $i$ caused by $\operatorname{ship} j$.

\section{Model verification}

\subsection{Experimental design}

In this paper, single ship type, of which the DWT is 70000 , the ship length is $230 \mathrm{~m}$, the ship breadth is $32.2 \mathrm{~m}$, is selected [8]. And two experiment groups are designed: $\mathrm{T} 1$, which is set on the paralleled traffic, is to analyze the variation of complexity caused by relative distance; T2, which is set on the "cross" intersection, is to analyze the variation of complexity caused by traffic conflict. The number of ships in $\mathrm{T} 1$ and T2 are 9 and 8 respectively, and the ship speed is set as $8 \mathrm{kn}$.

\subsection{Experimental results}

\subsubsection{Experiment T1}

In $\mathrm{T} 1$, the own-ship is in the location of $(0,0)$, ship course is 000 , the distance to the other-ship is 0.9 nmile, which is shown in fig.3(1). Fig.3(2) is the complexity distribution pattern in the initial moment. From fig.3(2) to (6), the time interval of the adjacent two pictures is $5 \mathrm{~min}$, and the solid line in red is the motion curve of the ownship. From these figures, we can see that shorter the distance between other-ship and own-ship is, higher the complex degree of the own-ship caused by the other-ship will be, which well reflected the relationship between traffic density and complexity.

\subsubsection{Experiment T2}

In T2, the own-ship is in the location of $(-4,0)$, ship course is 045 , distance between two adjacent ships is $0.85 \mathrm{n}$ mile. The course of the traffic flow in the left side of the own-ship is 045 , and that in the right side is 315 . And ships in both side are symmetry about the $\mathrm{Y}$ axis. Fig.4(1) is the complexity distribution pattern in the initial moment, and from fig.4(2) to (6), the time interval is $10 \mathrm{~min}$.

In fig.4(2) and (3), we can see that ships in the portside are close to the own-ship, therefore, the first ship in the portside has comparatively higher complexity, other ships are in the same course with the own-ship and there exists no converging tendency, so their complexity is lower. Ships in the starboard has encounter tendency with the own-ship, but their TCPA is large, so the urgency degree is low. Fig.4(4) to (6) show that as time goes on, ship density and complex degree in the portside change little, also has a little change. But in the starboard, the influence of the traffic conflict factors is obvious. In fig.4(4), when $\mathrm{t}=20 \mathrm{~min}$, as DCPA of the first ship in the starboard is small, its complex degree is higher than other ships, so the own-ship should stay alert. In fig.4(5), when $t=30 \mathrm{~min}$, complexities between the own-ship and ships in both side have exceed 50, and actions should be taken. As time 
goes on, complexities of ships in the starboard keep increase, and when $\mathrm{t}=40 \mathrm{~min}$ (shown in fig.4(6)), the first ship in the starboard collides with the own ship, and the complexity reaches its maximum value. Experiment T2 reflected the variation of complexity with the changing of traffic conflict, it is related with whether the converging tendency exists and the converging consequences.

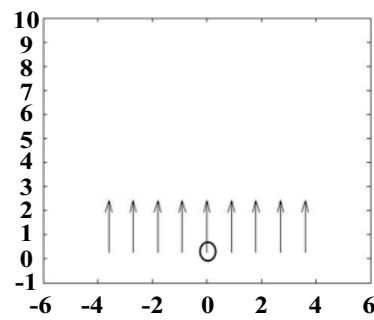

(1)

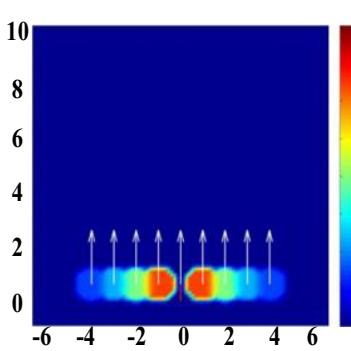

(3)

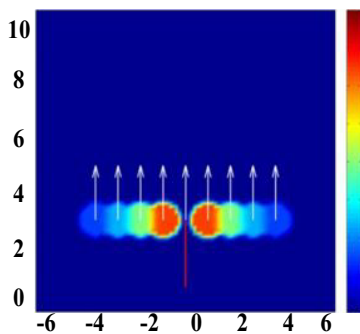

(5)

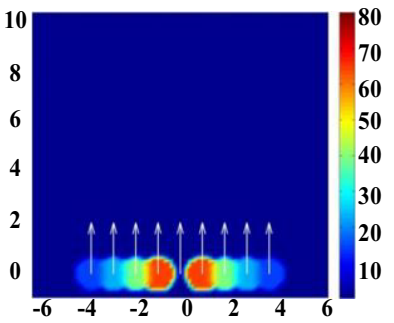

(2)

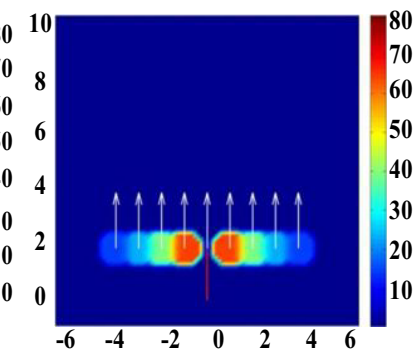

(4)

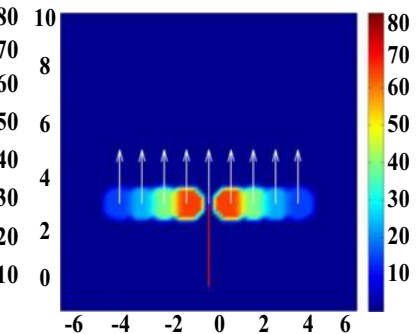

(6)
Figure 3. The spatial complexity distribution in parallel traffic.

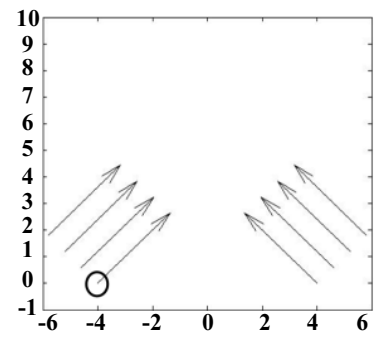

(1)

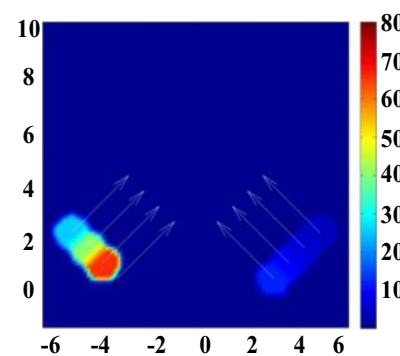

(3)

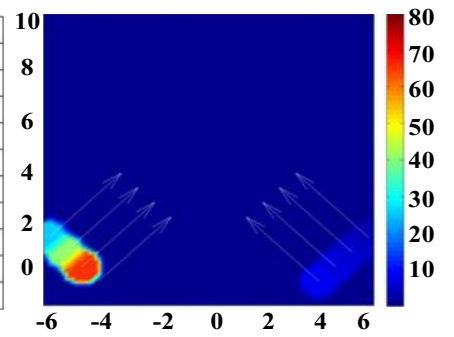

(2)

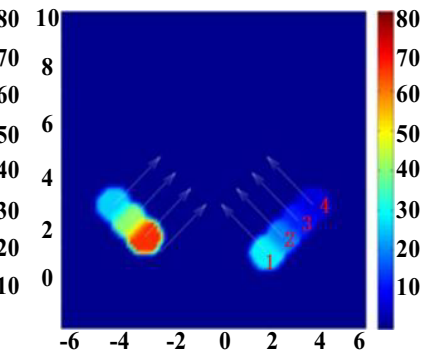

(4)

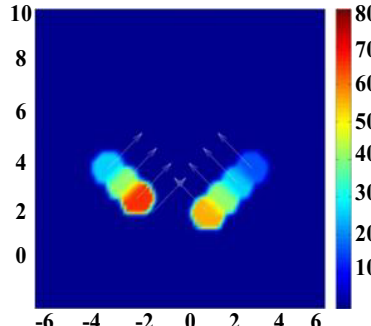

(5)

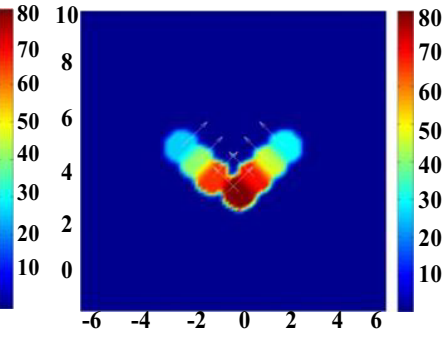

(6)
Figure 4. The spatial complexity distribution in "Cross" intersection traffic.

From $\mathrm{T} 1$ and $\mathrm{T} 2$, we can see that using marine traffic situation complexity model, the following two points can be realized: On the one aspect, traffic density factor can be used to recognize the dangerous degree of the surrounding traffic environment, and risk level of othership can be labeled. On the other aspect, traffic conflict factor can be used to recognize the surrounding ships which have potential conflicts with the own-ship or may cause operational constraints to the own-ship.

\section{4 conclusions}

In this paper, the marine traffic situation compleity, which is closely related with the traffic flow characteristics, is introduced to describe the complicated situation of marine traffic. Traffic flow is characterized by the vessel traffic density in the area as well as the geometrical relationships between vessels. Therefore, researches on the traffic situation complexity can be carried from two aspects, one is the vessel traffic density, the other is the vessel traffic conflict caused by the taffic situation. Then based on the analysis of marine traffic density and traffic conflict, the mathematical model of marine traffic situation complexity is established on the ideal condition. To verify the influences of different traffic conflict factors on the traffic situation complexity, senario simulations of different traffic structures are made. The experimental results demonstrated that: The model of marine traffic situation complexity proposed in this paper can well reflect the influences of the traffic density and traffic conflict on the marine traffic situation complexity. Not only the ships with collision risks can be identified, but ships with potential risks can also be recognized. Research in this paper can be a good basis for the future research of the security of the marine traffic system.

However, in this paper, influences of the mandatory rules (stipulated by Regulations for Preventing Collisions) and human factors on the traffic complexity are not considered, and influences of the ship types and channel conditions are not discussed as well. Therefore, how to describe the influences of these factors through mathematical expressions, and to establish the more universal model of marine traffic situation complexity need further research.

\section{Acknowledgement}


This work is supported by the National Science Foundation of China (NSFC) (No. 51579204), and is financially supported by self-determined and innovative research funds of WUT (No: 2016IVA064).

\section{References}

1. Y.Q. WEN, H. ZHANG, P. WAN, Risk degree analysis of ship collision risk in channel based on ordered probit model, J. China Safety Science Journal. 22, 134-139 (2012).

2. LAUDEMAN I. V., SHELDEN S. G., BRANSTROMR, et al: Dynamic density: An air traffic management metric, R. NAS 1.15:112226, NASA/TM-1998-112226. USA Springfield: NTIS (1998).

3. CHATTERJI G. B., SRIDHAR B., Measures for air traffic controller workload prediction, C. // 1st AIAA Aircraft, Technology Integration, and Operations Forum. USA. Los Angeles: AIAA Press (2001).
4. F.Q. DAI, L.S. HONG, Study on Airspace Traffic Complexity Computation Methods, J. Journal of Civil Aviation University of China. 28(3), 1-4 (2010). (In Chinese)

5. DELAHAYE D., PUECHMOREL S., Air traffic complexity based on dynamical systems, C. // 2010 49th IEEE Conference on Decision and Control (CDC), Atlanta: 0743-1546978-1-4244-7745-6.

6. C. ZHANG, J. ZHANG, M.H. HU, Air traffic complexity based on alliance effects, C. // The 28th Digital Avionics Systems Conference. USA Seattle: IEEE/AIAA Press (2009).

7. B.J. YE, M.H. HU, C. ZHANG, et-al, Traffic structure based air traffic complexity modeling, J. Journal of Transportation Systems Engineering and Information Technology. 12(1), 166-172 (2012). (In Chinese)

8. A.M. DU, Study on port fairway capacity based on standard vessels, D. Dalian: Dalian University of Technology (2008). 